Artículos Científicos

\title{
Modelo pedagógico de integración sinérgica para la enseñanza de las ciencias experimentales
}

\author{
Pedagogical Model of Synergistic Integration for the Teaching of Experimental \\ Sciences
}

\begin{abstract}
Modelo pedagógico de integração sinérgica para o ensino de ciências experimentais
\end{abstract}

\author{
Mireya Monroy Carreño \\ Instituto Politécnico Nacional, México \\ m.monroy449@gmail.com \\ https://orcid.org/0000-0002-3611-8532 \\ Ignacio Enrique Peón Escalante \\ Instituto Politécnico Nacional, México \\ ignaciopeon@gmail.com \\ https://orcid.org/0000-0002-8857-5735
}

\section{Resumen}

En un mundo cada vez más globalizado, con un mayor número de avances científicos y tecnológicos, se requiere que los estudiantes tengan una formación científica que les permita estar a la vanguardia de las necesidades actuales. Por ende, es necesario dejar de lado los paradigmas tradicionales y posicionar en su lugar modelos que integren diversos enfoques que se ajusten a la complejidad del contexto. El objetivo de este estudio es, en primer lugar, revisar modelos pedagógicos que contribuyan a mejorar el aprendizaje de las asignaturas que conforman el área de ciencias experimentales, y en segundo, proponer un modelo pedagógico de integración sinérgica que permita promover un proceso de enseñanza-aprendizaje que sea consistente con los requerimientos de la sociedad actual y con ello propiciar el interés de los estudiantes hacia el estudio de estas áreas. 


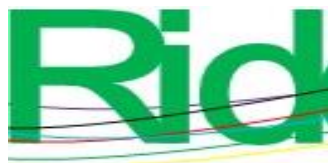

Revista Iberoamericana para la Investigación y el Desarrollo Educativo

ISSN $2007-7467$

Para alcanzar lo anterior se utilizó la metodología de sistemas suaves de Checkland, que estudia a los sistemas flexibles que poseen un alto componente social, cultural y político, como lo es el ámbito educativo, y obtener así una propuesta cimentada en los modelos constructivista, social-cognitivo, pensamiento complejo y ecológico contextual con un enfoque eco-cibersistémico-transdisciplinario. Esta investigación es parte de una primera etapa que incluye la visión holística de la enseñanza de las ciencias experimentales. Posteriormente se realizará un estudio de caso en el Colegio de Ciencias y Humanidades plantel Vallejo.

Palabras clave: aprendizaje, ciencias, complejidad, proceso de enseñanza.

\section{Abstract}

In an increasingly globalized world, with greater number of scientific and technological advances, students require to have a scientific background that allows them to be at the forefront of current needs. Therefore, it is necessary to replace traditional paradigms by models that integrate different approaches that fit the complexity of the context. The objective of this study is, first, to review pedagogical models that contribute to improving the learning of the subjects that make up the area of experimental sciences, and second, to propose a pedagogical model of synergistic integration that allows to promote a teaching-learning process that is consistent to the requirements of today's society and thereby foster the interest of students towards the study of these areas.

For this, the Checkland soft systems methodology was used to study flexible systems that have a high social, cultural and political component, such as the educational field, thus obtaining a model based on the constructivist, social-cognitive, complex thinking models and ecological context with an eco-cyber-systemic-transdisciplinary approach. This research is part of a first stage that includes the holistic vision of the teaching of experimental sciences. Later a case study will be carried out at the Colegio de Ciencias y Humanidades Campus Vallejo.

Keywords: learning, science, complexity, teaching process. 


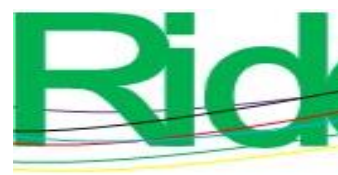

\section{Resumo}

Em um mundo cada vez mais globalizado, com um número maior de avanços científicos e tecnológicos, os alunos precisam ter uma formação científica que lhes permita estar na vanguarda das necessidades atuais. Portanto, é necessário deixar de lado paradigmas tradicionais e modelos de posição que integram diferentes abordagens que se encaixam na complexidade do contexto. $\mathrm{O}$ objetivo deste estudo é, em primeiro lugar, revisar modelos pedagógicos que contribuam para melhorar a aprendizagem das disciplinas que compõem a área das ciências experimentais e, em segundo lugar, propor um modelo pedagógico de integração sinérgica que permita promover um processo de ensino. aprendizado que seja consistente com os requisitos da sociedade atual e, assim, fomente o interesse dos alunos pelo estudo dessas áreas.

Para tanto, foi utilizada a metodologia de sistemas flexíveis Checkland, que estuda sistemas flexíveis com alto componente social, cultural e político, como o campo educacional, e obtém, assim, uma proposta baseada nos modelos sociais construtivistas. pensamento contextual cognitivo, complexo e ecológico, com uma abordagem ecossistêmica-sistêmica-transdisciplinar. Esta pesquisa faz parte de uma primeira etapa que inclui a visão holística do ensino experimental de ciências. Posteriormente, um estudo de caso será realizado na Escola de Ciências e Humanidades Vallejo.

Palavras-chave: aprendizagem, ciência, complexidade, processo de ensino.

Fecha Recepción: Julio 2019

Fecha Aceptación: Diciembre 2019

\section{Introducción}

Debido a que en los últimos años se han realizado diversas investigaciones para conocer los factores que han ocasionado una crisis en la educación científica y que se ha encontrado que, si bien los orígenes del alejamiento de la ciencia y la tecnología en México son diversos, uno de los elementos más relevantes está centrado en la enseñanza de la educación básica (Flores, 2012), este estudio se centra en la enseñanza y en el aprendizaje de las asignaturas que conforman el área de ciencias experimentales (ACE).

La enseñanza de la ciencia en el ámbito educativo presenta problemas debido al uso de estrategias pedagógicas tradicionales que no fomentan la comprensión de los temas científicos y tecnológicos (López, 2015), y provocan así una crisis al no poder responder a los nuevos retos sociales. Puesto que, como bien es sabido, existe una diferencia entre la enseñanza de la ciencia en 


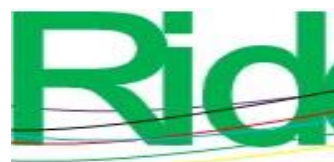

Revista Iberoamericana para la

Investigación y el Desarrollo Educativo

ISSN $2007-7467$

las aulas y lo que realmente tiene que saber el estudiante para la toma de decisiones, lo cual ha originado conocimientos fragmentados o descontextualizados y, por consiguiente, una visión poco globalizada. Como resultado se han obtenido elevadas cifras de analfabetismo de la ciencia en el mundo occidental (Matthews, 2017).

Lo anterior se manifiesta en el informe del Programa Internacional para la Evaluación de Estudiantes [PISA] (Organización para la Cooperación y el Desarrollo Económicos [OCDE], 2015), donde los resultados muestran que solo $8 \%$ de los alumnos de los países que integran la OCDE alcanzan los niveles más altos de competencia con relación a la ciencia. En México está realidad no es diferente, ya que únicamente $0.1 \%$ de los participantes consiguen los mismos niveles, y esto no se ha modificado desde el 2006, lo que significa que $48 \%$ de los jóvenes en México no poseen el mínimo de los conocimientos científicos que le permitan participar en una sociedad compleja (Instituto Nacional para la Evaluación de la Educación [INEE], 2016).

Así, pues, resulta evidente que se requieren modificar los modelos de aprendizaje de las ciencias y proponer estrategias activas (Arteaga, Armada y Del Sol, 2016) que desarrollen habilidades cognitivas, instrumentales y transversales en los alumnos, con el fin de extender el aprendizaje a su entorno (Drăghicescu, Petrescua, Cristeab, Gorghiuc y Gorghiuc, 2014). No obstante, la mayoría de los centros educativos no se ha desprendido de la enseñanza tradicional (Pantoja y Covarrubias, 2013).

En esa misma línea, Reimers y Chung (2016) exponen que debe existir un cambio pedagógico y de valoración con el objetivo de propiciar un aprendizaje autodirigido del mundo real; sin embargo, como ya se mencionó, todavía muchos espacios educativos siguen implementando sistemas de enseñanza tradicional; sistemas que han demostrado no permitir el desarrollo de competencias científicas demandadas en el contexto actual en la mayoría de las personas (Sanmartí y Márquez, 2017).

Sin duda, en un mundo cada vez más globalizado y con nuevas exigencias que cumplir, se requiere formar individuos con conocimientos, habilidades, actitudes y valores que les permitan comprender y transformar su entorno. Al mismo tiempo, la formación científica es conveniente no solo porque de ella depende que los estudiantes se sientan o no atraídos por carreras relacionadas con ciencias; para numerosos alumnos será la última oportunidad de adquirir la cultura necesaria a fin de entender el desarrollo científico y tecnológico que se desenvuelve a su alrededor, lo que también le permitirá ejercer como ciudadano crítico y responsable del siglo XXI (Secretaría de Educación Pública [SEP], 2017). 


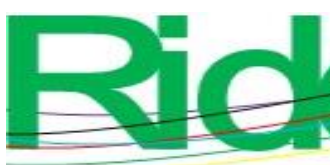

Revista Iberoamericana para la

Investigación y el Desarrollo Educativo

ISSN $2007-7467$

Partiendo de los supuestos anteriores, el objetivo de este estudio es revisar modelos pedagógicos que contribuyan a mejorar el aprendizaje de las asignaturas que conforman el ACE a nivel medio superior, particularmente del Colegio de Ciencias y Humanidades (CCH), y así proponer un modelo pedagógico de integración sinérgica (MPIS) que permita promover un proceso de enseñanza-aprendizaje que sea consistente con los requerimientos de la sociedad actual y que, además, sea capaz de propiciar el interés de los estudiantes hacia el estudio de estas áreas.

\section{Habilidades requeridas en la sociedad del conocimiento}

La educación, en términos generales, necesita desarrollar las habilidades que requiere el ciudadano del siglo XXI; de entre las cuales destaca el pensamiento científico y el análisis crítico. Ello con el fin de proporcionar las bases para que los individuos participen en temas relacionados con la ciencia y tecnología de manera instruida, dado que si es ajeno a este tipo de conocimiento será incapaz de intervenir y, en consecuencia, se verá en la necesidad de delegar a otras personas su responsabilidad.

Con respecto a lo anterior, la figura 1 muestra las habilidades que se necesitan promover en el alumno. Cabe destacar que estas no son nuevas, e incluso probablemente fueron solicitadas o utilizadas en el pasado; no obstante, a diferencia de las épocas anteriores, no están enfocadas a un grupo selecto, sino que son requeridas para toda la población (Reimers y Chung, 2016).

Figura 1. Habilidades necesarias para la población en el siglo XXI

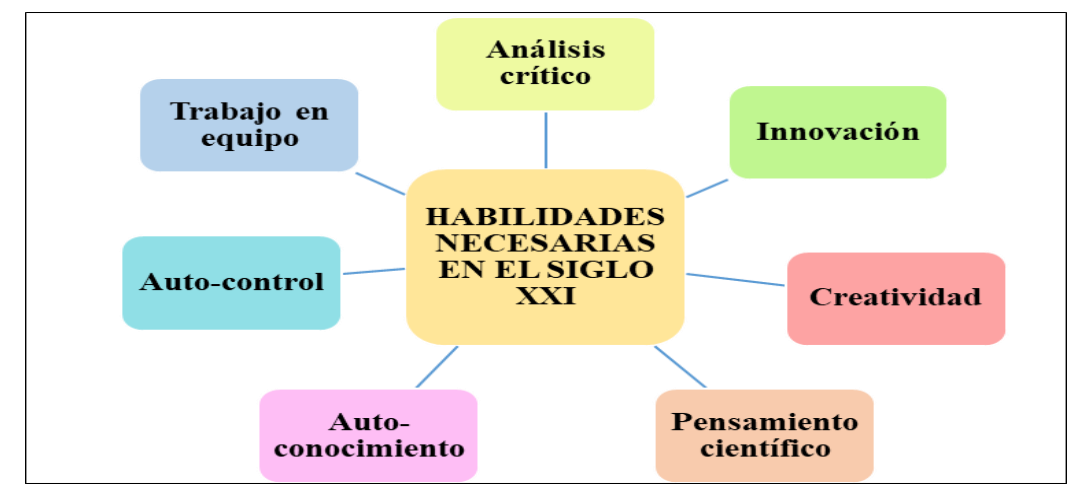

Fuente: Elaboración propia con base en Reimers y Chung (2016) 


\section{Propósitos de la enseñanza de las ciencias}

La enseñanza de la ciencia ha tenido diversos propósitos a lo largo de la historia. Al inicio se estudiaba porque era requisito para ingresar a la educación superior. Posteriormente, su intención fue la formación de científicos con el fin de cubrir las demandas de los desarrollos tecnológicos de la época (De Freitas Dias y Alves, 2010). En los últimos años, esta perspectiva se transformó, dado que se le dio mayor importancia a la enseñanza relacionada con contextos de la vida cotidiana que incluyan cuestiones sociales y tecnológicas; en el desarrollo de una educación científica básica que permita formar ciudadanos activos y responsables (Adúriz et. al, 2011).

En la actualidad, el estudio de las asignaturas que conforman el ACE tiene la responsabilidad de promover conocimientos en los alumnos que respondan a los procesos y procedimientos complejos del mundo real, por lo tanto, hoy en día, la enseñanza de la ciencia en el nivel medio superior posee numerosas finalidades para atender las necesidades de la sociedad actual (ver figura 2).

Figura 2. Los principales propósitos de le enseñanza de la ciencia

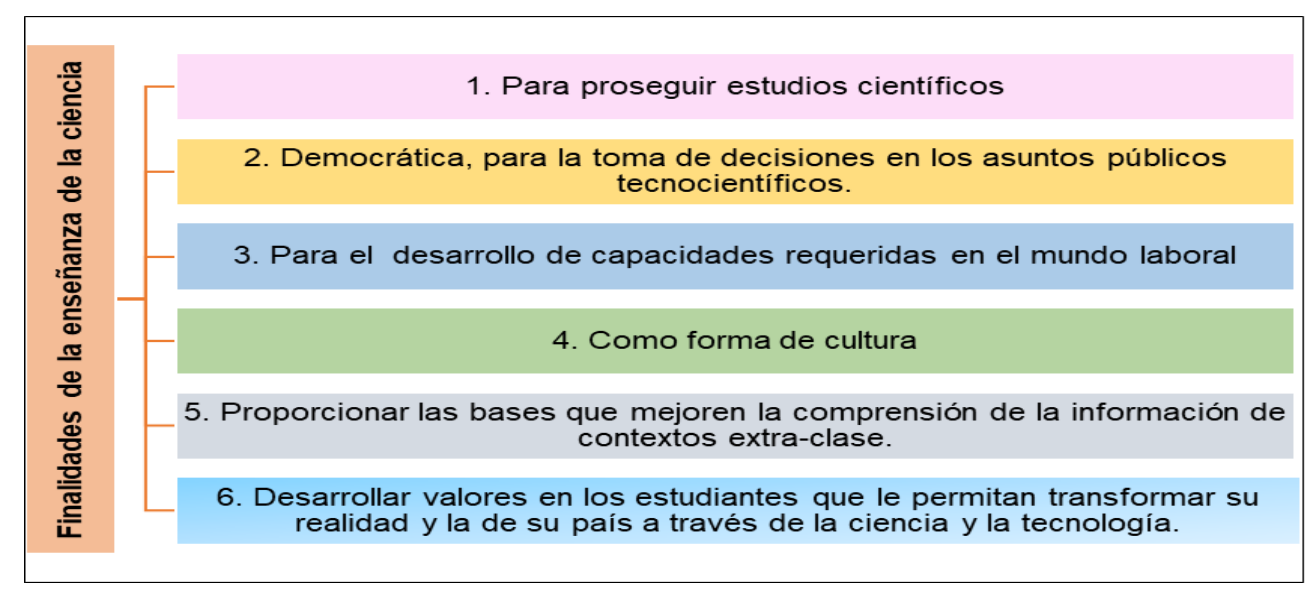

Fuente: Elaboración propia con base en Arteaga et al. (2016)

Cabe agregar que los objetivos de la enseñanza de ciencia en ámbitos preuniversitarios deben corresponder al entorno personal, local y global del estudiante, como se expresa en la figura 3. La importancia de esto radica en que el alumno comprenda su propia cultura y con ello desarrolle un pensamiento crítico, reflexivo y propositivo. 
Figura 3. Finalidades de la enseñanza de la ciencia en el contexto personal, local y global

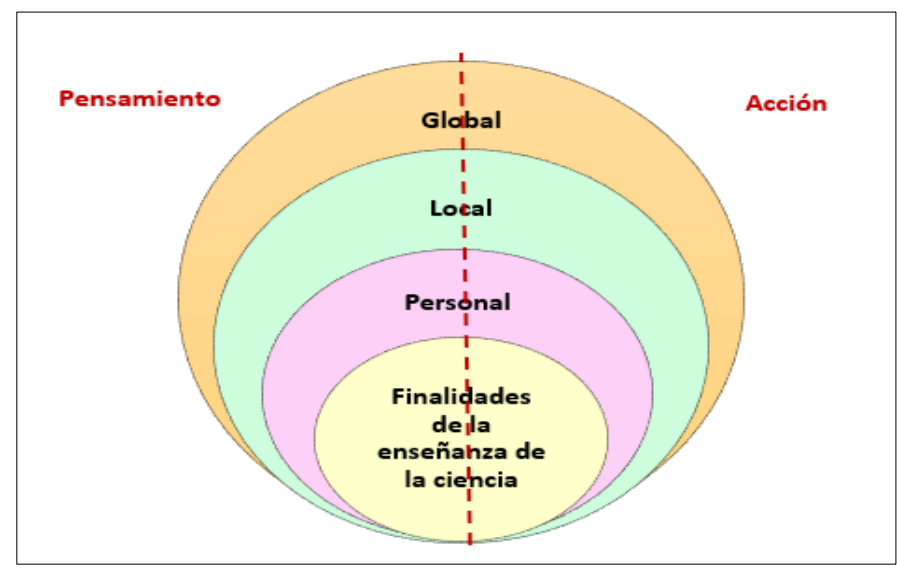

Fuente: Elaboración propia

\section{Modelo pedagógico}

Un modelo pedagógico es la representación esquemática, sistemática y conscientemente simplificada del conjunto de relaciones que describen un fenómeno con el fin de facilitar su comprensión (Abarca, 2007). Asimismo, es un esquema teórico en el que se detalla qué se debe enseñar, qué debe de aprender el estudiante, cómo aprende el alumno, la metodología de enseñanza, la evaluación de los conocimientos, define el rol de los docentes y los estudiantes, así como las interacciones entre ellos (Bournissen, 2014, p. 248).

Los modelos pedagógicos están compuestos de aspectos psicológicos del proceso de aprendizaje, sociológicos, comunicativos, ecológicos o gnoseológicos (Ortiz, 2011). Sobre la base de las ideas arriba expuestas se puede decir que un modelo pedagógico es un planteamiento integral e integrador referente a cierto fenómeno desde un punto de vista teórico y práctico (Vergara y Cuentas, 2015, p. 915).

Por otro lado, un modelo pedagógico "establece los parámetros para analizar las prácticas del acto pedagógico que se convierte en un proceso permanente de construcción social y en un aspecto investigativo cotidiano que exige recopilación de información y sistematizarla para su posterior análisis e interpretación” (Vives, 2016, p. 42). En la misma orientación, los modelos pedagógicos pueden promover cambios en la estructura de la clase, en los contenidos, métodos, objetivos, actividades y evaluación. Al mismo tiempo, son una guía oculta hacia el desarrollo de tareas formativas (García y Buitrago, 2017). 


\section{Metodología}

Este estudio está enfocado en la metodología de sistemas suaves de Checkland (véase figura 4). Esta se basa en emplear partes del mundo real como sistemas con el fin de hallar una solución a situaciones problemáticas de manera flexible, a causa de que cada contexto es único y demanda una metodología que se ajuste a las características y necesidades de cada situación, ya que en la realidad un mismo suceso no se repite dos veces del mismo modo (Checkland, 2013).

Figura 4. Metodología para sistemas blandos

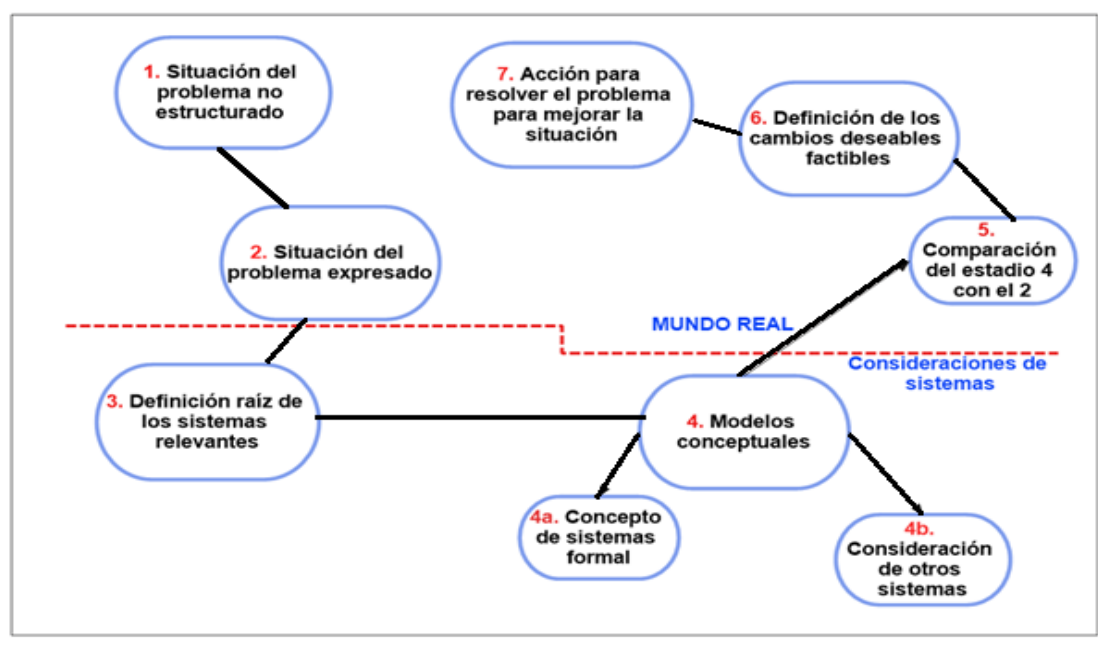

Fuente: Checkland y Scholes (1990)

El esquema anterior se fragmenta en dos partes, integradas por las fases 1, 2, 5, 6 y 7, las cuales están inmersas en el mundo real. Las fases 3 y 4 están incluidas en el pensamiento sistémico. A continuación, se define brevemente en qué consistió cada una de las partes de la metodología de Checkland.

1) Situación del problema no estructurado: Esta investigación se centra en el $\mathrm{CCH}$ plantel Vallejo, por lo tanto, en este estadio se recopiló información por medio de una investigación documental y la aplicación de una encuesta a 24 profesores y 92 alumnos de dicha institución, acerca de cuáles son las dificultades de la enseñanza de las ciencias experimentales.

2) Situación del problema expresado: Con respecto a la información recabada en el paso número uno, se construyó el esquema de las principales dificultades que existen en la enseñanza de las ciencias.

3) Definición raíz: Esta cimentada en seis factores CATWOE que hace referencia a los clientes, actores, proceso de transformación, weltanschauung (visión del mundo), propietario del sistema y entorno. 


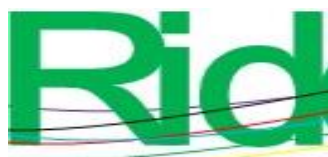

4) Modelos conceptuales: Partiendo de la definición raíz se expone cómo se debería de enseñar la ciencia.

5) Comparación del estadio cuatro con el dos: Con base en la información de cómo se debería enseñar la ciencia y cuáles son sus finalidades a nivel medio superior, se procedió a revisar los modelos pedagógicos que, por sus características teóricas o prácticas, podrían mejorar la enseñanza de la ciencia.

6) Definición de los cambios deseables: En este estadio se eligieron las particularidades de los modelos pedagógicos que se examinaron en el paso anterior en función de lo que se espera como proceso y producto de le enseñanza de la ciencia.

7) Acción para resolver el problema: En este paso se diseñó el modelo pedagógico integrado que contempla las características elegidas de los modelos pedagógicos revisados en la fase anterior.

Con respecto a la situación no estructurada del problema, es necesario precisar que el modelo pedagógico propuesto por el $\mathrm{CCH}$ tiene un enfoque constructivista, de carácter propedéutico y de cultura básica. Está orientado a la formación intelectual y social de sus estudiantes, considerados sujetos de la cultura y de su propia educación. Asimismo, prioriza los aprendizajes a alcanzar por los alumnos por medio de contenidos conceptuales, procedimentales y actitudinales, con la finalidad de explicar los fenómenos naturales.

Desafortunadamente, las materias del ACE ocupan el segundo lugar en deserción e índice de reprobación en el $\mathrm{CCH}$; solo son superadas por las asignaturas que conforman el área de matemáticas.

En la tabla 1 se muestra los resultados de la investigación documental, con respecto a las problemáticas que existen en la enseñanza de las asignaturas del ACE. 
Tabla 1. Dificultades en la enseñanza de las ciencias experimentales

\begin{tabular}{|c|c|}
\hline Autor & Dificultades en la enseñanza de las ciencias experimentales \\
\hline Adúriz et al. (2011) & $\begin{array}{l}\text { El uso de libros en las clases, cuya función principal es leer su } \\
\text { contenido escrito. }\end{array}$ \\
\hline $\begin{array}{l}\text { Dávila, Borrachero, Cañada, } \\
\text { Martínez y Sánchez (2015) }\end{array}$ & $\begin{array}{l}\text { Impartir contenidos científicos } \\
\text { mayoritariamente emociones negativas. }\end{array}$ \\
\hline $\begin{array}{l}\text { Furman, Luzuriaga, Taylor, } \\
\text { Anauati y Podestá (2018) }\end{array}$ & $\begin{array}{l}\text { Repetición mecánica y memorización de hechos, definiciones } \\
\text { y algoritmos (p. 83). }\end{array}$ \\
\hline Garzón y Pérez (2015) & $\begin{array}{l}\text { No se considera el lenguaje como medio de comunicación de } \\
\text { los saberes, los cuales son alejados del contexto social y } \\
\text { cultural de los estudiantes. }\end{array}$ \\
\hline González (2015) & $\begin{array}{l}\text { Carencia de los conocimientos a enseñar y el desconocimiento } \\
\text { de cómo enseñarlos (p. 18). }\end{array}$ \\
\hline Martínez y Riveros (2019) & Experimentos aislados y descontextualizados. \\
\hline Pantoja y Covarrubias (2013) & Predomina la transmisión de los conocimientos. \\
\hline $\begin{array}{l}\text { Torres (1975; citado en Ortega } \\
\text { y Gil, 2018), Solbes et al. } \\
\text { (2007; citados en Ortega y Gil, } \\
\text { 2018) y Vílchez et al. (2015; } \\
\text { citados en Ortega y Gil, 2018) }\end{array}$ & $\begin{array}{l}\text { La aplicación de evaluaciones cuantitativas dirigidos a } \\
\text { calificar el contenido memorizado y no otras formas de } \\
\text { aprendizaje. }\end{array}$ \\
\hline $\begin{array}{l}\text { Padilla, Brooks, Jiménez y } \\
\text { Torres (2016). }\end{array}$ & $\begin{array}{l}\text { Desarticulación del currículo entre las asignaturas que } \\
\text { conforman el del área de ciencias (p. 17). }\end{array}$ \\
\hline López (2015) & $\begin{array}{l}\text { Desafíos del entorno en aspectos contextuales en relación con } \\
\text { las características de los estudiantes, el medio educativo, los } \\
\text { fines, propósitos y valores de la enseñanza (p. 76). }\end{array}$ \\
\hline Macedo (2016) & $\begin{array}{l}\text { Una enseñanza basada en conocimientos cerrados, acabados, } \\
\text { sin relación entre ellos y su realidad solo es en el ámbito } \\
\text { educativo. }\end{array}$ \\
\hline SEP (2017) & $\begin{array}{l}\text { La falta de interés, e incluso rechazo hacia el estudio de las } \\
\text { ciencias, asociado al fracaso escolar. }\end{array}$ \\
\hline
\end{tabular}

Fuente: Elaboración propia 


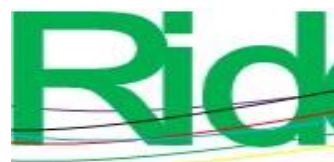

\section{Revista Iberoamericana para la Investigación y el Desarrollo Educativo \\ ISSN $2007-7467$}

Asimismo, con base en la información obtenida de la tabla 1 se elaboró un instrumento de evaluación (encuesta) con la finalidad de conocer la percepción de los actores del proceso de enseñanza-aprendizaje, en particular con respecto a las dificultades que presentan las materias del ACE en cuatro aspectos: 1) contenido, 2) actividades, 3) evaluación y 4) actitudes; para lo cual se desarrolló una técnica de muestreo no probabilístico de tipo consecutivo para 24 profesores del área de experimentales (8 profesores de química, 10 de física y 6 de biología) y 92 alumnos entre las edades de 14 a 18 años (44 mujeres y 48 hombres). Las respuestas obtenidas se sintetizaron y se muestran en la tabla 2. 
Tabla 2. Percepción de los profesores y de los alumnos encuestados del CCH plantel Vallejo sobre las dificultades de la enseñanza de las materias de ciencias experimentales

\begin{tabular}{|c|c|c|}
\hline Criterios & Profesores & Alumnos \\
\hline & $\begin{array}{l}\text { - Dificultades para contextualizar algunos } \\
\text { contenidos del programa de estudio. } \\
\text { - Hay que abordar demasiados contenidos en } \\
\text { un lapso muy corto. } \\
\text { - Es complicado relacionar los aprendizajes } \\
\text { con el resto de las asignaturas que integran el } \\
\text { ACE, debido a las características de cada } \\
\text { programa de estudio. }\end{array}$ & $\begin{array}{l}\text { - Algunos temas no tienen utilidad en } \\
\text { el entorno personal. } \\
\text { - Las clases de ciencias } \\
\text { experimentales son interesantes, no } \\
\text { obstante, son difíciles por el uso de } \\
\text { las matemáticas, las unidades y los } \\
\text { conceptos que se deben aplicar. }\end{array}$ \\
\hline 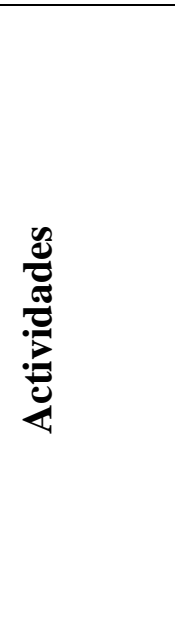 & $\begin{array}{l}\text { - La falta de materiales complica la } \\
\text { realización de actividades experimentales. } \\
\text { - Por cuestiones de seguridad, se evitan hacer } \\
\text { experimentos o solo se hacen algunos muy } \\
\text { sencillos. } \\
\text { - Las actividades deben ser simples para que } \\
\text { las terminen en clase, de lo contrario no las } \\
\text { hacen de tarea. }\end{array}$ & $\begin{array}{l}\text { - Se realizan pocos experimentos en } \\
\text { clase. } \\
\text { - Los maestros principalmente hacen } \\
\text { experimentos demostrativos y no } \\
\text { entiendo cuál es propósito de la } \\
\text { actividad. } \\
\text { - Los profesores son poco dinámicos, } \\
\text { ya que las actividades se centran en } \\
\text { leer y en exponer. }\end{array}$ \\
\hline 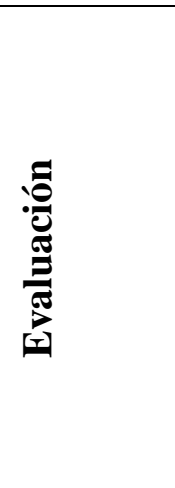 & $\begin{array}{l}\text { - Por cuestiones de tiempo solo se } \\
\text { promueven evaluaciones cuantitativas. } \\
\text { - En las evaluaciones diagnósticas se observa } \\
\text { que existen errores conceptuales por parte de } \\
\text { los alumnos. }\end{array}$ & $\begin{array}{l}\text { - Las evaluaciones no son acordes } \\
\text { con lo que el maestro enseñó en } \\
\text { clase. } \\
\text { - Los maestros son los únicos que } \\
\text { evalúan. } \\
\text { - Desconocen la forma de evaluación } \\
\text { del curso. }\end{array}$ \\
\hline
\end{tabular}




\begin{tabular}{|l|l|l|}
\hline & $\begin{array}{l}\text { - Los alumnos no les interesan las clases de } \\
\text { ciencias, existe una falta de compromiso por }\end{array}$ & - Los profesores se enojan si se les \\
preguntan las dudas. \\
sus estudios. & - Los profesores no les interesan si \\
& - Los estudiantes tienen una predisposición & entendemos, sino que entreguemos \\
negativa hacia las materias de ciencias & los trabajos. \\
experimentales. & \\
\hline
\end{tabular}

Fuente: Elaboración propia

Desde una perspectiva más general, se identificaron cuáles eran las situaciones problemáticas que tenían mayor incidencia obteniendo así la figura 5.

Figura 5. Esquema de la situación problemática de la enseñanza de las ciencias

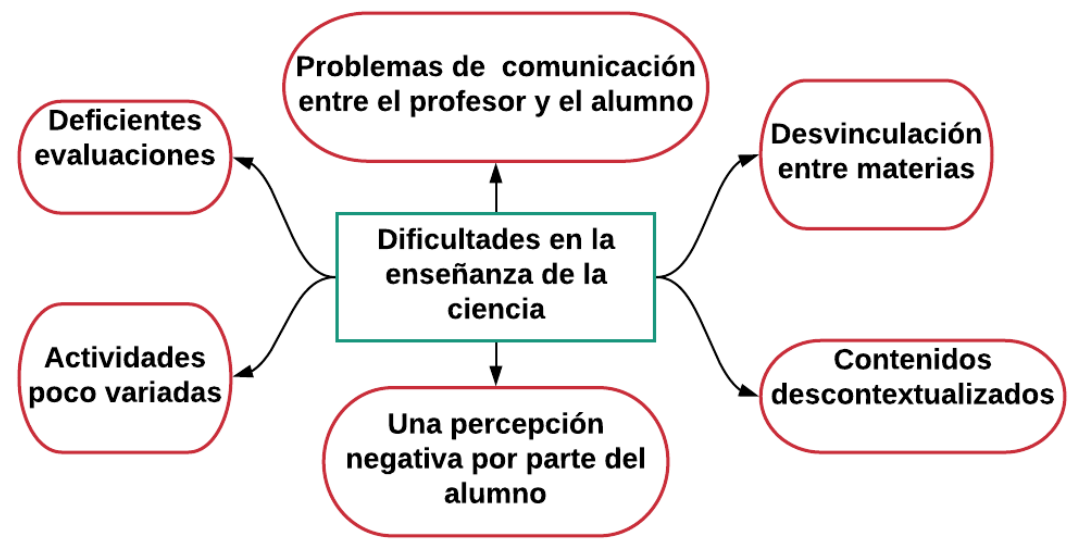

Fuente: Elaboración propia

Con base en lo anterior, la definición raíz para este sistema es el diseño de un modelo pedagógico que vincule los conocimientos racionales emergentes con la dimensión emocional, considerando los factores exógenos y endógenos del contexto, con el fin de propiciar la inclusión del pensamiento científico-sistémico, crítico, propositivo y activo y lograr así aprendizajes significativos en el estudiante para que los implementen en su vida personal y en los ambientes local y global. Cabe destacar que la definición raíz se fundamentó en el CATWOE que se describe a continuación:

- $\quad$ Cliente (C): El beneficiario principal es el alumno. Los beneficiarios indirectos serán los docentes, padres de familia, la comunidad del CCH y la sociedad.

- $\quad$ Actores (A): Compañeros, profesor, padres de familia, escuela, comunidad y sociedad. 


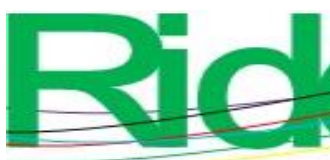

\section{Revista Iberoamericana para la Investigación y el Desarrollo Educativo \\ ISSN $2007-7467$}

- Transformación (T): Identificar las entradas, el proceso y la salida, las cuales se muestran en la tabla 3.

Tabla 3. Proceso de transformación

\begin{tabular}{|c|c|c|}
\hline Entrada & Proceso & Salida \\
\hline $\begin{array}{l}\text { Una enseñanza basada } \\
\text { en conocimientos } \\
\text { cerrados, aislados, } \\
\text { descontextualizados y } \\
\text { fragmentados. }\end{array}$ & $\begin{array}{l}\text { Una propuesta pedagógica híbrida en la que } \\
\text { se integren las fortalezas de cada modelo, } \\
\text { con la finalidad de abarcar un mayor } \\
\text { número de factores que afectan la } \\
\text { enseñanza y el aprendizaje en ciencias } \\
\text { experimentales. }\end{array}$ & $\begin{array}{l}\text { Una enseñanza } \\
\text { contextualizada, } \\
\text { transdisciplinaria, } \\
\text { vivencial y } \\
\text { práctica. }\end{array}$ \\
\hline
\end{tabular}

Fuente: Elaboración propia

- Weltanschauung (W): Permita promover un proceso de enseñanza-aprendizaje que sea consistente con los requerimientos de la sociedad actual y con ello propiciar el interés de los estudiantes hacia el estudio del ACE.

- $\quad$ Propietario del sistema (O): Profesores y autoridades del $\mathrm{CCH}$.

- $\quad$ Entorno (E): Es la Universidad Nacional Autónoma de México (UNAM), según sus lineamientos, contexto cultural, social y político que envuelven el $\mathrm{CCH}$.

Partiendo de la definición raíz, se consideró cómo se debería de enseñar la ciencia para mejorar los resultados obtenidos hasta ahora (ver tabla 4). 
Tabla 4. Como se debería de enseñar la ciencia

\begin{tabular}{|c|c|}
\hline Autor & Cómo se debería de enseñar la ciencia \\
\hline Torres $(2010)$ & $\begin{array}{l}\text { Los docentes requieren una didáctica coherente y adecuada al } \\
\text { actual contexto sociocultural (p. 140) }\end{array}$ \\
\hline Dávila et al. (2015) & $\begin{array}{l}\text { El docente debe favorecer y promover emociones positivas para } \\
\text { inducir y estimular el aprendizaje. }\end{array}$ \\
\hline Adúriz et al. (2011) & $\begin{array}{l}\text { Planificar una enseñanza de las ciencias en la que las ideas, los } \\
\text { procesos, las capacidades y las actitudes se complejizan, y se } \\
\text { generan nuevas formas de participación en las diversas } \\
\text { actividades. }\end{array}$ \\
\hline $\begin{array}{l}\text { Bravo, Ramírez, Faúndez y } \\
\text { Astudillo (2016) }\end{array}$ & $\begin{array}{l}\text { Para motivar, desarrollar el pensamiento científico y las } \\
\text { habilidades asociadas es necesario implementar un gran número de } \\
\text { actividades experimentales (p. 106). }\end{array}$ \\
\hline $\begin{array}{l}\text { De Freitas Dias y Alves } \\
(2010)\end{array}$ & $\begin{array}{l}\text { Contextualización de la enseñanza de la ciencia, considerando los } \\
\text { intereses y necesidades de aquellos que aprenden (p. 278). }\end{array}$ \\
\hline Padilla et al. (2016). & Clases más prácticas y vivenciales. \\
\hline Macedo (2016) & $\begin{array}{l}\text { Una formación de educadores de ciencias que permita cambiar las } \\
\text { maneras de enseñar, a partir de la indagación, de situaciones de } \\
\text { problemas abiertos y contextualizados (p. 14). }\end{array}$ \\
\hline Ortega y Gil (2018) & Enseñar con un enfoque que enfatice la ciencia y la tecnología. \\
\hline SEP (2017) & $\begin{array}{l}\text { Establecer una relación más explícita con el medio natural y con la } \\
\text { sociedad. }\end{array}$ \\
\hline Arteaga et al. (2016) & $\begin{array}{l}\text { Desarrollar formas de actividad y de comunicación colectivas que } \\
\text { favorezcan la interacción de lo individual con lo colectivo. }\end{array}$ \\
\hline Serna (2015) & $\begin{array}{l}\text { Para hacer ciencia hoy es necesario tener mente abierta, interactuar } \\
\text { con múltiples disciplinas e inmiscuirse en problemas complejos (p. } \\
50 \text { ). }\end{array}$ \\
\hline
\end{tabular}

Fuente. Elaboración propia

Con base a las consideraciones anteriores, pues, se estudiaron modelos pedagógicos que de manera conjunta puedan mejorar el proceso de enseñanza, para tales efectos se excluyeron los modelos tradicionales y se centró la investigación en aquellos que consideran al alumno como el protagonista del proceso educativo, una educación contextualizada y con énfasis en el aprendizaje, 


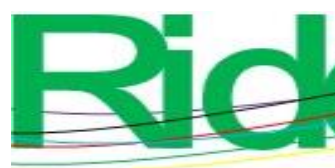

Revista Iberoamericana para la

Investigación y el Desarrollo Educativo

ISSN $2007-7467$

es decir, que tenga un mismo punto de partida, la razón de esto es que si dos modelos intentan explicar el mismo fenómeno de la realidad no son incongruentes (Galagovsky y Adúriz, 2001), no obstante, cada uno tiene ciertas características que lo hacen único e interesante. A continuación, se mencionan y se explica por qué se eligieron dichos modelos.

- Constructivismo: Considera los conocimientos previos del estudiante, sus experiencias e intereses con el fin de aplicarlos a su entorno personal, local y global (Sanmartí y Márquez, 2017). De esta manera, convierte al estudiante en el protagonista del proceso educativo, siempre por medio del desarrollo del pensamiento analítico, crítico, creativo que promueva la construcción del conocimiento y se oriente hacia la ciencia (Pantoja y Covarrubias, 2013).

- Social cognitivo: La fuente social se considera de especial relevancia en la definición de los propósitos de la enseñanza de la ciencia. Lo anterior a causa de que es indispensable proponer un currículo relacionado con las necesidades sociales para evitar rupturas entre el mundo y la escuela (SEP, 2017). Asimismo, la enseñanza de las ciencias tiene el deber de preparar al ser humano para la vida y esto se logra no solo a través de los conocimientos, sino permitiendo la búsqueda del conocimiento a partir de ambientes problemáticas tomadas del entorno, donde se pueda valorar las posibilidades de aplicación de la ciencia en la vida (Arteaga et al., 2016).

- Pensamiento complejo: Hoy en día la ciencia es dinámica, compleja y transdisciplinaria, ya que los problemas son integrales, lo que implica que se requieren soluciones que respondan a estas necesidades (Serna, 2015). Por tal motivo, se requiere abordar desde la complejidad que no solo se centra en la enorme cantidad de interacciones entre los elementos sino incluye la incertidumbre (García y Buitrago, 2017).

- Ecológico contextual: Este paradigma facilita y apoya la asimilación y conceptualización de los aspectos ambientales que lo rodea, y provoca así una conciencia de respeto por el contexto que lo rodea y el medio ambiente (Trujillo, 2017). De acuerdo con Torres (2010), plantea que la ciencia debería permitir a los habitantes del planeta conseguir un ambiente sano y en equilibrio, con el objetivo de construir un mundo sostenible y con justicia; para ello se deben desarrollar valores y que den las pautas de cómo intervenir en los contextos individuales, la sociedad y el medio ambiente de manera responsable e informada (Adúriz et al., 2011, p.15).

La figura 6 muestra el MPIS que surge a partir de la necesidad de proponer una solución integral desde diferentes enfoques que envuelva el mayor número de factores que afectan a la enseñanza de la ciencia y dé origen así a un modelo híbrido. Siguiendo esta vez a Menéndez y 


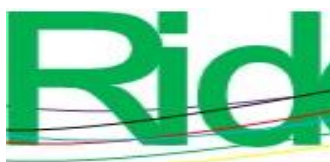

Revista Iberoamericana para la

Investigación y el Desarrollo Educativo

ISSN $2007-7467$

Fernández (2016, p. 115), esta puede ser una opción interesante en el campo educativo al permitir que el proceso de enseñanza-aprendizaje se beneficie de las ventajas que cada modelo proporciona.

Figura 6. Modelos pedagógicos que integran el MPIS

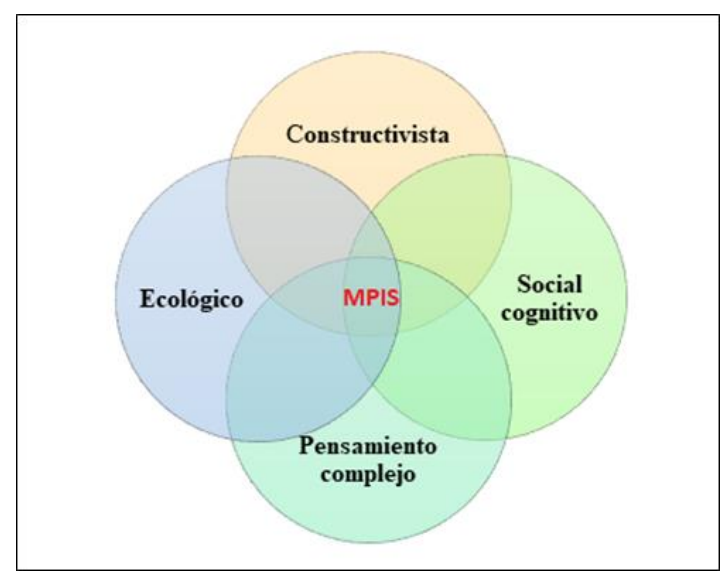

Fuente: Elaboración propia

Ahora bien, teniendo en cuenta las bases anteriores, es posible explicar brevemente las ventajas y desventajas de cada modelo planteado líneas arriba:

- Constructivismo: Este modelo tiene una gran presencia en la actualidad, dado que en el proceso educativo se consideran las concepciones previas de los estudiantes, su entorno, interacción con los compañeros de clase, proporcionan cabida al error como método para construcción de conocimientos universalmente válidos (Vergara y Cuentas, 2015, p. 930), con todo lo cual se promueve una actitud proactiva.

En el mismo sentido, visualiza al alumno como responsable de la construcción de su propio aprendizaje, en el que se integran entornos a ejes globalizados conceptuales, procedimentales y actitudinales, donde el aprendizaje es una construcción para modificar la estructura mental y lograr niveles altos de complejidad, integración y diversidad (Flórez, 1999), lo que produce un aprendizaje flexible.

○ Ventajas: Desarrolla un aprendizaje desde lo más complejo a lo más sencillo, favorece la coordinación de experiencias motoras, lo que le permite desarrollar una cognición superior (Vargas y Jiménez, 2014); así, genera un mayor desarrollo intelectual en los alumnos, sin dejar a un lado que es un modelo que considera el aspecto social en la educación (Méndez, Villalobos, D’Alton, Cartín y Piedra, 2012, p.48). 


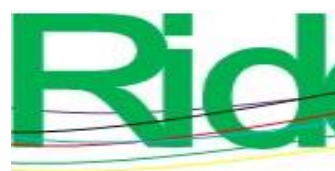

Revista Iberoamericana para la

Investigación y el Desarrollo Educativo

ISSN $2007-7467$

○ Desventajas: Estimula el individualismo, ya que es encaminada al objeto de estudio, el cual es utilizado como plataforma para la construcción de su conocimiento y problemas epistemológicos, dado que no explica cuál es el papel del docente y cómo este influye en el alumno, puesto que el constructivismo parte del hecho de que el estudiante construye por sí solo el conocimiento (Vargas y Jiménez, 2014).

Cabe agregar que es un modelo pedagógico que le falta un enfoque científico consistente y unificado del desarrollo cognitivo, además de que le da una gran prioridad al lenguaje como principal herramienta, lo que implica que se requiera un alto nivel de conocimiento lingüístico por parte del docente. Asimismo, el constructivismo se perfila como una opción costosa por los recursos, el tiempo y de difícil realización (Méndez et al., 2012, p. 48).

- Social cognitivo: Este modelo hoy en día está presente en los procesos de enseñanzaaprendizaje ya que permite que tanto el proceso como la evaluación sean dinámicos, lo que hace que el aprendizaje sea significativo en los alumnos (Vergara y Cuentas, 2015, p.933). Además, el aprendizaje depende del contexto social y cultural de los estudiantes, el cual se construye a partir de interacciones sociales, lo cual está vinculado con la zona de desarrollo próximo propuesta por Vygotsky.

El modelo social cognitivo desarrolla habilidades e intereses en los alumnos en función del contexto social y cultural, centrándose en la realidad, la teoría y la praxis, promueve un trabajo productivo, contextualizado en problemas, promueve el rol entre docente y alumno de forma horizontal y bidireccional (Flórez, 1999).

○ Ventajas: Desarrolla habilidades e intereses de los alumnos con relación en su contexto social y cultural que propicie el conocimiento científico (Flórez, 1999), promueve la motivación intrínseca, se plantean objetivos por capacidades (procesos cognitivos), valores (procesos afectivos) (Trujillo, 2017, p. 58). Aunado a lo anterior, los temas o problemáticas son planteadas de una realidad concreta y se trabajan de manera integral con la misma comunidad (Vives, 2016).

○ Desventajas: El estudiante aprende a efectuar una tarea sin que sea la forma adecuada de hacerlo. Es posible que no exista motivación inicial y se visualiza en una sola dirección y desde un solo enfoque. Asimismo, es un modelo que implica demasiado tiempo.

- Pensamiento complejo: Las principales características de este modelo es que comprende al ser humano de forma integral. Considera que el pensamiento no es reduccionista ni totalizante sino reflexivo. Para ello, el conocimiento debe ser contextualizado, globalizado, 


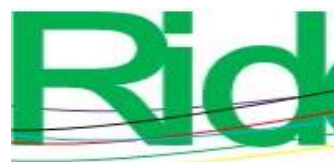

Revista Iberoamericana para la

Investigación y el Desarrollo Educativo

ISSN $2007-7467$

dinámico y emergente, el cual se construye y no se da por transferencia. El rol del docente es considerar al ser humano de forma integral (Morin, 1999).

Cabe mencionar que este paradigma está orientado a la reconstrucción del sujeto, del conocimiento, del mundo y la vida, dado que los sistemas de ideas, teorías y el conocimiento son dinámicos, por lo que resulta que solo es posible permitir que cada alumno construya, busque y sea responsable de su propio proceso formativo (García y Buitrago, 2017, p. 121).

Dentro de este mismo orden de ideas, este modelo considera que el aprendizaje es un fenómeno emergente que involucra diversos procesos (neuronales, corporales, emocionales y ambientales), los cuales no pueden ser reducidos solo a uno de sus componentes, por ello se considera que el aprendizaje es inesperado y, en la mayoría de los casos, imposible de prever, y que el contenido es al mismo tiempo simple y complejo (Serna, 2015, p. 119).

- Ventajas: Promueve en los estudiantes el pensamiento reflexivo, crítico y propositivo, busca la formación integral de los estudiantes, estudia el fenómeno educativo desde diferentes perspectivas, integral de diversas disciplinas, deja de lado el pensamiento reduccionista.

○ Desventajas: Considera la incertidumbre, lo cual propicia visiones erróneas de la realidad, lo que produce a su vez obstáculos epistemológicos (Serna, 2015).

- $\quad$ Ecológico contextual: Este modelo parte del hecho de que el conocimiento se construye y se complementa con lo que aportan los estudiantes, por ende, requiere estudiar las interacciones persona-grupo-medio ambiente, dado que el proceso de enseñanza-aprendizaje depende de factores personales, psicosociales y de contexto. Y así es capaz de generar en el alumno una actitud activa, reflexiva, innovadora, crítica y de investigación; de ahí que incorpora al contexto personal, familiar, escolar, local y global de los estudiantes desde una perspectiva holística, global, con criterio planetario, colectivo y sustentable (Trujillo, 2017).

○ Ventajas: Considera el proceso de enseñanza-aprendizaje interactivo y continuo, incluye procesos no observables como son los pensamientos, creencias, percepciones y actitudes (Trujillo, 2017).

○ Desventajas: Gimeno y Pérez (2008, p. 135) mencionan que es un paradigma que se centra en el análisis de lo que sucede en el aula descuidando la parte teológica de toda actividad educativa, y quizás es un modelo que desestime la importancia de los contenidos y experiencias de aprendizaje al centrarse en los intereses y preocupaciones de los alumnos, los cuales están alejados de los contenidos y experiencias que constituyen el currículo oficial. 


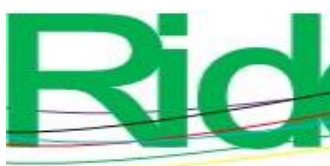

\section{Revista Iberoamericana para la Investigación y el Desarrollo Educativo \\ ISSN $2007-7467$}

En función de lo planteado, se proponen los cambios y las acciones que se necesitan implementar con el fin de mejorar el proceso de enseñanza aprendizaje (véase tabla 5).

Tabla 5. Cambios y acciones para propiciar una mejor enseñanza y aprendizaje en ciencias experimentales

\begin{tabular}{|c|c|}
\hline Cambios & Acciones \\
\hline $\begin{array}{l}\text { Desarrollar habilidades y capacidades que le } \\
\text { permitan a los alumnos construir nuevos } \\
\text { conocimientos científicos por cuenta propia, } \\
\text { conocimientos que le ayuden a comprender su } \\
\text { realidad para descartar información } \\
\text { seudocientífica o creencias. }\end{array}$ & $\begin{array}{l}\text { Enseñar a partir de situaciones problemáticas } \\
\text { abiertas y contextualizadas, considerando la } \\
\text { incertidumbre de la realidad. }\end{array}$ \\
\hline $\begin{array}{l}\text { El estudiante debe de tener la capacidad de } \\
\text { integrar los contenidos de cada una de sus } \\
\text { asignaturas, con la finalidad de aplicarlas en su } \\
\text { entorno personal, social o profesional. }\end{array}$ & $\begin{array}{l}\text { Enseñar la ciencia desde problemáticas } \\
\text { actuales y de interés social, que se aborde a } \\
\text { partir de diferentes visiones, considerando } \\
\text { experimentos prácticos y teóricos para } \\
\text { proponer soluciones integrales y propiciando } \\
\text { un enfoque transdisciplinario. }\end{array}$ \\
\hline $\begin{array}{l}\text { Contribuir a la formación de individuos con } \\
\text { valores que propicien el pensamiento crítico, } \\
\text { reflexivo y propositivo. }\end{array}$ & $\begin{array}{l}\text { Promover estrategias activas, participativas y } \\
\text { creativas que estimulen el progreso del alumno. }\end{array}$ \\
\hline Promover un conocimiento pertinente. & $\begin{array}{l}\text { Situar una enseñanza en contexto, a causa de } \\
\text { que la ciencia es un producto de la sociedad, de } \\
\text { la cultura y del entorno. }\end{array}$ \\
\hline $\begin{array}{l}\text { Propiciar emociones positivas en los alumnos } \\
\text { con respecto a las asignaturas que conforman } \\
\text { el área de experimentales. }\end{array}$ & $\begin{array}{l}\text { Atender la parte afectiva, emocional, social, } \\
\text { cultural, psíquica y cognitiva de los } \\
\text { estudiantes. }\end{array}$ \\
\hline $\begin{array}{l}\text { Formar un estudiante interesado en aspectos } \\
\text { políticos, ambientales, económicos, entre } \\
\text { otros, y que participe de manera activa, } \\
\text { informada y con valores. }\end{array}$ & $\begin{array}{l}\text { Considerar el desarrollo de los estudiantes a } \\
\text { partir de los diversos ambientes en los que } \\
\text { desenvuelve y que pueden influir en el cambio } \\
\text { del progreso cognitivo, relacional, actitudinal y } \\
\text { moral }\end{array}$ \\
\hline
\end{tabular}




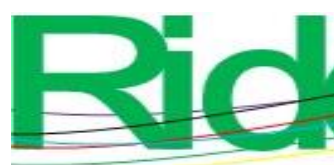

Revista Iberoamericana para la Investigación y el Desarrollo Educativo

ISSN 2007 - 7467

\begin{tabular}{|l|l|}
\hline Las evaluaciones son integrales, permanentes & $\begin{array}{l}\text { Promover la heteroevaluación, coevaluación; } \\
\text { y dinámicas considerando el contexto y los } \\
\text { propósitos académicos. }\end{array}$ \\
& $\begin{array}{l}\text { favorecer las autoevaluaciones con la finalidad } \\
\text { de los alumnos conozcan sus fortalezas y } \\
\text { sus debilidades. }\end{array}$ \\
\hline
\end{tabular}

Fuente: Elaboración propia

\section{Resultados}

De acuerdo con el análisis procedente, se propone el MPIS, el cual está conformado por cuatro holones o niveles, lo que hace referencia a que son parte de un sistema global. A continuación, se explica brevemente cada holón o nivel (ver figura 7):

- $\quad$ Primer holón: Es el punto de partida del modelo, considerando a los alumnos como el centro del proceso de aprendizaje, puesto que se debe identificar los conocimientos, habilidades, actitudes que poseen los estudiantes para integrarlas al proceso enseñanza-aprendizaje, ya que para lograr aprendizajes significativos es necesario relacionar el ámbito escolar y el entorno con la finalidad de que el estudiante perciba la utilidad del conocimiento adquirido y así propiciar una motivación intrínseca.

- $\quad$ Segundo holón: Incluye las habilidades que se pretenden lograr con los estudiantes: aprender-aprender, aprender-ser, aprender-hacer, aprender-convivir y aprender-emprender. Y que estas estén en función del contexto social, cultural, ecológica y tecnológica del alumno, considerando que hoy en día se reclama una educación que contribuya al progreso de un país, ya que suscita el desarrollo comercial, industrial y, en términos generales, a mejorar la calidad de vida.

- $\quad$ Tercer holón: Hace referencia a grandes rasgos de las características del modelo pedagógico (metas, contenido, desarrollo, método, relación profesor-alumno y evaluación).

- $\quad$ Cuarto holón: Corresponde a los contextos en que se desenvuelve los estudiantes según la teoría ecológica de los sistemas de Bronfenbrenner, en donde se jerarquiza el contexto y se divide en los siguientes niveles: microsistemas, que están conformados por las relaciones que tienen contacto directo con el estudiante (compañeros, profesor, padres de familia, escuela); mesosistema, que son las interconexiones que comprenden dos o más entornos en los que participan activamente los alumnos (familia, amigos, vida social, entre otros); exosistema, son las interconexiones que se dan entre los medios en los que el individuo no participa activamente, pero en los que se originan hechos o decisiones que perturban directamente al estudiante (comunidad, 


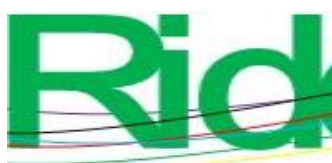

\section{Revista Iberoamericana para la Investigación y el Desarrollo Educativo ISSN $2007-7467$}

sistema educativo, medios de comunicación, redes sociales), y el macrosistema, que está interconectado con las características de la sociedad y de su cultura (sociedad, cultura, normas, entre otras).

\section{Figura 7. MPIS}

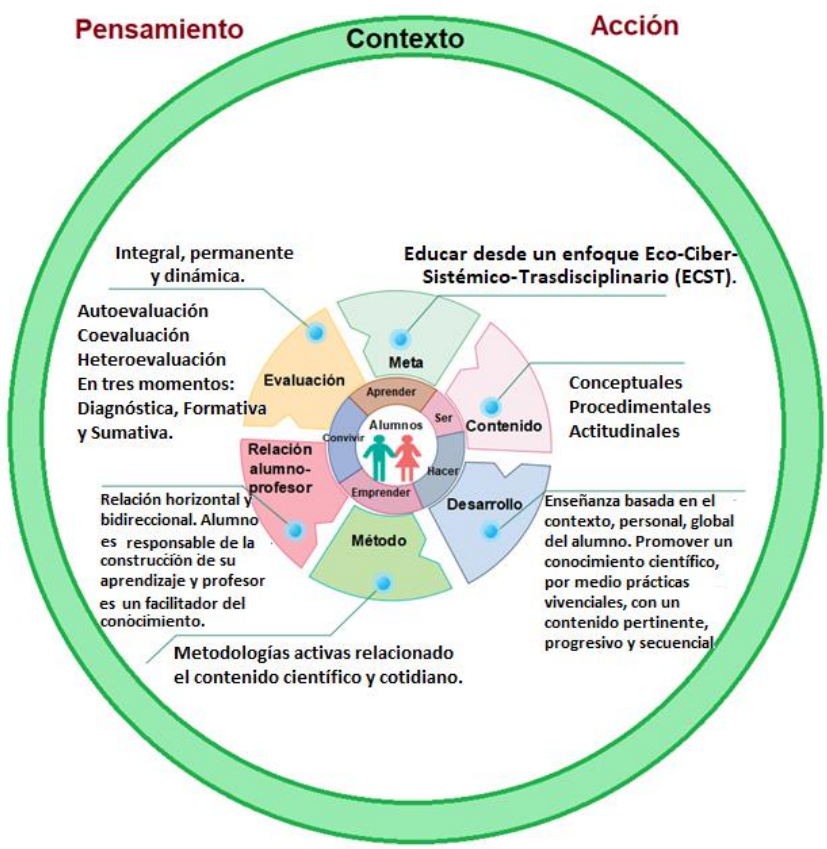

Fuente: Elaboración propia

El modelo MPIS está centrado en métodos activos y participativos para el desarrollo integral; parte del hecho de que los alumnos poseen conocimientos de tipo racional, emocional, empírico e intuitivo que se deben organizar de una manera gradual, didáctica y complementaria y así lograr un mayor impacto en el aprendizaje.

Cabe agregar que el MPIS tiene un enfoque eco-ciber-sistémico-transdisciplinario, puesto que integra el pensamiento, la acción y la cultura, lo que da lugar a la transdisciplinariedad; y si se asume que el proceso educativo está constituido por sistemas biológicos, abiertos, dinámicos y sociales, en los que intervienen procesos de control y comunicación, lo cual en el ámbito educativo tiene la intención de mejorar el proceso comunicativo e informativo entre el alumno, los compañeros, los recursos didácticos y el profesor a través de la retroalimentación, entonces se alude a la cibernética.

Asimismo, se deben contemplar las relaciones del microsistema, mesosistema, macrosistema y exosistema de los participantes que vinculan el contexto natural y civilizatorio; en 
otras palabras, incluir el conocimiento ecológico en el desarrollo del proceso enseñanzaaprendizaje para la adecuada construcción del conocimiento (ver figura 8).

Figura 8. Enfoque eco-ciber-sistémico-transdisciplinario

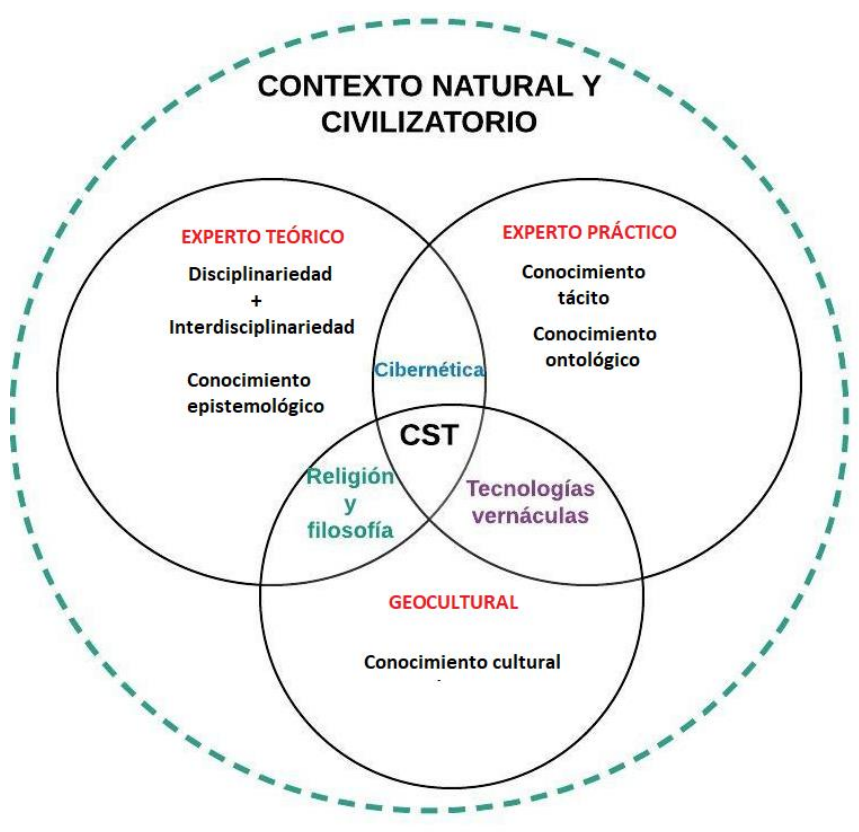

Fuente: Elaboración propia con base en Licona, Ortega, Hernández y Peón (2017)

\section{Discusión}

La enseñanza de las ciencias en el nuevo milenio demanda de profundas transformaciones desde la educación elemental hasta la universidad, de modo que el profesor deje de ser un transmisor de conocimientos acabados y tome conciencia de que su labor es crear las posibilidades para que el alumno produzca y construya su conocimiento (Arteaga et al., 2016).

Sobre las ideas anteriores es necesario propiciar una educación integral a partir de diferentes enfoques pedagógicos que conlleven a modelos híbridos, es decir, la combinación de dos o más orientaciones, puesto que "no existe un modelo pedagógico único u omnipotente que solucione todos los problemas de aprendizaje que tienen los estudiantes" (Ortiz, 2011, p.135); debido, además, a que cada modelo pedagógico tiene sus ventajas pero también sus debilidades, por lo que no permiten ser aplicados totalmente (Flórez, 1999, p. 55).

Con base en las consideraciones anteriores, los modelos deben de modificarse a partir de los avances científicos y tecnológicos, ya que, conforme a Galagovsky y Adúriz (2001), estos son construcciones provisorias y perfectibles. Asimismo, de acuerdo con Ortiz (2011), "es primordial 


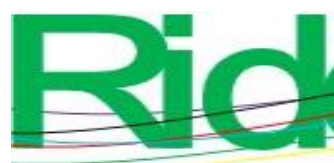

Revista Iberoamericana para la

Investigación y el Desarrollo Educativo

ISSN $2007-7467$

que en cualquier concepción que se asuma, se haga desde una mirada más holística, compleja, dialéctica y sistémica del pensamiento" (p. 130), con la finalidad de que potencialice al máximo las habilidades de los estudiantes para generar aprendizajes aplicables a situaciones complejas (Abarca, 2007).

Una de las fortalezas de este modelo es que integra las particularidades de modelos pedagógicos ya probados y en los cuales se han obtenidos buenos resultados. La finalidad de proponer el MPIS es que envuelva un mayor número de aspectos que puedan mejorar los efectos obtenidos hasta ahora en el ACE. La limitación de esta investigación es que es una propuesta teórica que está en función de lo que se espera que logren los estudiantes. Y si se retoman las palabras de González (2015, p. 17): si aprender ciencias es difícil, entonces enseñar ciencias tampoco es una tarea fácil.

\section{Conclusión}

La propuesta de este MPIS no pretende reemplazar ningún modelo, sino proponer una alternativa integral en la cual se utilicen las características y cualidades de diferentes modelos pedagógicos que, de manera conjunta, puedan abarcar un mayor número de factores que intervienen en el proceso educativo. Si bien es complicado que un modelo aborde todas las variables que existen en un salón de clase, esto no significa que los modelos deban de estancarse; al contrario, estos tienen que evolucionar conforme a los requerimientos de la sociedad.

Debe señalarse que todo el desarrollo del presente estudio se centró en las dificultades, las finalidades y en cómo debería de ser la enseñanza de las ciencias, para así elegir las características de cada modelo que, al integrarse, le proporcionen una identidad al MPIS; pero debe dejarse de lado la implementación para otra fase de la investigación: Un estudio de caso en el CCH y así poder conocer sus alcances y sus limitaciones.

Finalmente, una línea para futuras investigaciones se podría orientar a estudiar modelos, paradigmas, técnicas, métodos, entre otros recursos, que han sido empleados en ambientes educativos, y evaluar qué tan efectivos serían si se combinan, cuáles serían sus limitaciones y sus alcances si se trabajan de manera conjunta. 


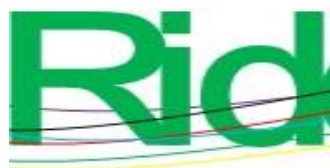

\section{Referencias}

Abarca, R. R. (2007). Modelos pedagógicos educativos de excelencia. Arequipa, Perú: Universidad Católica de Santa María.

Adúriz, A., Gómez, A. A., Rodríguez, D. P., López, D. M. Jiménez, M. P., Izquierdo, M. y Sanmartí, N. (2011). Las ciencias naturales en Educación Básica: formación de ciudadanía para el siglo XXI (1. a ed.). Ciudad de México, México: SEP.

Arteaga, E., Armada, L., y Del Sol, J. L. (2016). La enseñanza de las ciencias en el nuevo milenio. Retos y sugerencias. Revista Universidad y Sociedad, 8(1), 169-176.

Bournissen, J. M. (2014). Modelo pedagógico virtual. Signos Universitarios, anejo 2, 1, 245-266.

Bravo, A. A, Ramírez, G. P., Faúndez, C. A. y Astudillo, F. (2016). Propuesta didáctica constructivista para la adquisición de aprendizajes significativos de conceptos en física de fluidos. Formación universitaria, 9(2), 105-114.

Checkland, P. and Scholes J. (1990). Soft systems methodology in action. Chichester, England: Wiley.

Checkland, P. (2013). Soft Systems Methodology. In Gass, S. I. and Fu, M. C. (eds.), Encyclopedia of Operations Research and Management Science (1430-1436). Boston, Estados Unidos: Springer.

Dávila, M. A., Borrachero A. B., Cañada, F., Martínez, G. y Sánchez, J. (2015). Evolución de las emociones que experimentan los estudiantes del grado de maestro en educación primaria, en didáctica de la materia y la energía. Revista Eureka sobre Enseñanza y Divulgación de las Ciencias, 12(3), 550-564.

De Freitas Dias, K. A. y Alves, A. (2010). Reflexiones sobre el papel de la contextualización en la enseñanza de ciencias. Enseñanza de las ciencias, 28(2), 275-284.

Drăghicescua, L. M., Petrescua, A. M., Cristeab, A. G., Gorghiuc, L. M. and Gorghiu, G. (2014). Application of Problem-Based Learning Strategy in Science lessons- Examples of Good Practice. Procedia - Social and Behavioral Sciences, 149(2014), 297-301.

Furman, M., Luzuriaga, M., Taylor, I., Anauati, M. V. y Podestá, M. E. (2018). Abriendo la "caja negra" del aula de ciencias: un estudio sobre la relación entre las prácticas de enseñanza sobre el cuerpo humano y las capacidades de pensamiento que se promueven en los alumnos de séptimo grado. Enseñanza de las ciencias, 36(2), 81-103.

Flores, F. (2012). La enseñanza de la ciencia en la educación básica en México. Ciudad de México, México: INEE. 


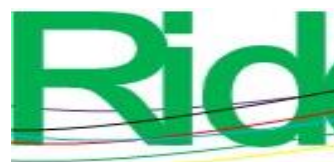

Revista Iberoamericana para la

Investigación y el Desarrollo Educativo

ISSN $2007-7467$

Flórez, R. (1999). Evaluación pedagógica y cognición. Bogotá, Colombia: McGraw-Hill.

Galagovsky, L. y Adúriz, A. (2001). Modelos y analogías en la enseñanza de la ciencia de las ciencias naturales el concepto de modelo didáctico analógico. Enseñanza de las ciencias, 19(2), 231-242.

García, M. S. y Buitrago, Y. C. (2017). Modelo pedagógico de pensamiento complejo en diseño gráfico. Revista Logos Ciencia y Tecnología, 8(2), 119-131.

Garzón, M. A. y Pérez, R. (2015). Lenguaje y los modelos en la enseñanza de la química. El caso de fenómeno químico. ACTAS, 3. Recuperado de http://filosofiaeducacion.org/actas/index.php/act/article/view/153.

Gimeno, J. y Pérez A. (eds.) (2008). La enseñanza: su teoría y su práctica. Madrid, España: Akal. González, F. (coord). (2015). Didáctica de las ciencias para la educación primaria. Madrid, España: Pirámides.

Instituto Nacional para la Evaluación de la Educación [INEE]. (2016). México en PISA 2015 (1. a ed.). México: INEE

Licona, J., Ortega, A., Hernández, L. y Peón, I. (2017). Diagnóstico sistémico transdisciplinar de las instituciones de educación superior promotoras de los derechos humanos de las mujeres. Revista de Teoría Educativa, 1(1), 31-43.

López, Z. (2015). La enseñanza de las ciencias naturales desde el enfoque de la apropiación social de la ciencia, la tecnología y la innovación ASCTI, en educación básica-media. Revista científica, 2(22), 75-84.

Macedo, B. (2016). Educación científica. Montevideo, Uruguay: Unesco.

Martínez, N. Y. y Riveros, S. Y. (2019). La enseñanza de caída libre bajo la metodología de aprendizaje activo. Tecné Episteme y Didaxis: TED, (45), 35-56.

Matthews, M. R. (2017). La enseñanza de la ciencia. Un enfoque desde la historia y la filosofía de la ciencia (1. ${ }^{\mathrm{a}}$ ed.). Ciudad de México, México: FCE.

Menéndez, J. I. y Fernández, J. (2016). Hibridación de los modelos de Educación Deportiva y Responsabilidad Personal y Social: una experiencia a través de un programa de kickboxing educativo. Retos. Nuevas Tendencias en Educación Física, Deporte y Recreación, (30), 113-121.

Méndez, V., Villalobos, A., D’Alton, C., Cartín, J. y Piedra, L. A. (2012). Los modelos pedagógicos centrados en el estudiante: apuntes sobre los procesos de aprendizaje y enseñanza. San José, Costa Rica: Programa de Investigación en Fundamentos de Educación a Distancia 


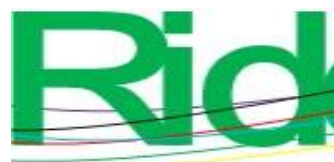

Revista Iberoamericana para la

Investigación y el Desarrollo Educativo

ISSN $2007-7467$

Morin, E. (1999). Los siete saberes para la educación del futuro. París, Francia: Unesco.

Organización para la Cooperación y el Desarrollo Económicos [OCDE]. (2015). Programa para la Evaluación Internacional de Alumnos (PISA) PISA-2015 - Resultados. Nota País/OCDE. Recuperado de https://www.oecd.org/pisa/PISA-2015-Mexico-ESP.pdf.

Ortega, V. y Gil, C. (2018). Estudio de aplicación de modelos didácticos de ciencias experimentales en un proyecto comunidad de aprendizaje. ReiDoCrea, 8, 80-94

Ortiz, A. (2011). Hacia una nueva clasificación de los modelos pedagógicos: el pensamiento configuracional como paradigma científico y educativo del siglo XXI. Praxis, 7(1), 121137.

Padilla, C., Brooks, P., Jiménez, L. D. y Torres, M. I. (2016). Dimensiones de las competencias científicas esbozadas en los programas de estudio de Biología, Física y Química de la Educación Diversificada y su relación con las necesidades de desarrollo científicotecnológico de Costa Rica. Revista Electrónica Educare, 20(1), 1-26.

Pantoja, J. C. y Covarrubias, P. (2013). La enseñanza de la biología en el bachillerato a partir del aprendizaje basado en problemas (ABP). Perfiles educativos, 35(139), 93-109.

Reimers, F. y Chung, C. K. (2016). Enseñanza y aprendizaje en el siglo XXI: metas, políticas educativas y currículo en seis países. Ciudad de México, México: FCE.

Sanmartí, N. y Márquez, C. (2017). Aprendizaje de las ciencias basado en proyectos: del contexto a la acción. Ápice. Revista de Educación Científica, 1(1), 3-16. Recuperado de https://doi.org/10.17979/arec.2017.1.1.2020.

Secretaría de Educación Pública [SEP]. (2017). Campo Disciplinar de Ciencias Experimentales Bachillerato General-Adecuación de contenidos de las asignaturas del componente de formación propedéutica básica. México: Secretaría de Educación Pública. Recuperado de https://bit.ly/30ZYxNZ.

Serna, E. (2015). Ciencia y pensamiento complejo - Desarrollo transdisciplinar de un paradigma (1. a ed.). Medellín, Colombia: Instituto Antioqueño de Investigación.

Torres, M. I. (2010). La enseñanza tradicional de las ciencias versus las nuevas tendencias educativas. Revista electrónica Educare, 14(1), 131-142.

Trujillo, L. M. (2017). Teorías pedagógicas contemporáneas. Bogotá, Colombia: Fundación Universitaria del Área Andina.

Vargas, C. y Jiménez, S.I. (2014). Constructivismo en los procesos de educación en línea. Revista Ensayos Pedagógicos, 8(2), 157-167.

\section{(CC)}


Vergara, G. y Cuentas H. (2015). Actual vigencia de los modelos pedagógicos en el contexto educativo. Opción, 31(6), 914-934.

Vives, M.P. (2016). Modelos pedagógicos y reflexiones para las pedagogías del Sur. Boletín Redipe, 5(11), 40-55.

\begin{tabular}{|c|c|}
\hline Rol de Contribución & Autor (es) \\
\hline Conceptualización & Mireya Monroy Carreño \\
\hline Metodología & Mireya Monroy Carreño (Igual) / Ignacio Enrique Peón Escalante (igual) \\
\hline Software & No aplica \\
\hline Validación & Mireya Monroy Carreño \\
\hline Análisis Formal & Mireya Monroy Carreño \\
\hline Investigación & Mireya Monroy Carreño \\
\hline Recursos & Mireya Monroy Carreño \\
\hline Curación de datos & Mireya Monroy Carreño (principal) / Ignacio Enrique Peón Escalante (apoya) \\
\hline $\begin{array}{l}\text { Escritura - Preparación del borrador } \\
\text { original }\end{array}$ & Mireya Monroy Carreño (principal) / Ignacio Enrique Peón Escalante (apoya) \\
\hline Escritura - Revisión y edición & Mireya Monroy Carreño (principal) / Ignacio Enrique Peón Escalante (apoya) \\
\hline Visualización & Mireya Monroy Carreño \\
\hline Supervisión & Mireya Monroy Carreño (igual) / Ignacio Enrique Peón Escalante (igual) \\
\hline Administración de Proyectos & Mireya Monroy Carreño \\
\hline Adquisición de fondos & Mireya Monroy Carreño \\
\hline
\end{tabular}

